[4] Stennbock H., Sent M. T. et Jones J. H.: Jour. Biol. Chem., Vol. LV, 4111923.

[5] Hess A.F. et UNGer L.J.: Jour. Biol. Chem., Vol. XXXVIII, 293, 1919.

[6] Hart E.B., Steenbock H. et Elurs N.R.: Jour. Biol. Chem., Vol. XLVI, $309,1921$.

[7] JoHnson J.M. et Hooper C.W.: Unites States Public Health Reports, Vol. XXXVII, 989, 1922.

[8] Jephcotт H. et Bacharach A.F. : Biochem. Jour., Vol. XV, 129, 1921.

]9] Supplee G.C. et Dow O.D.: American Journal of Diseases of Children Vol. XXXI, 41, 1926.

\title{
RECHERCHES SUR LA QUALITE DU LAIT A MONTPELLIER
}

Par F. CARRIEU,

Professeur Agrégé à la Faculté de Montpellier, Chế des Travaux d'Hygiène.

La qualité du lait, aliment de première nésessité pour l'enfant et le malude, a toujours interessé le médecin et l'hygiéniste. Dès 1906, on s'était préoccupé de la provenance ainsi que de la valeur alimentaire du lait consommé à Montpellier et l'on trouvera la réponse à ces deux questions dans le travail très complat de MM. BerTin-Sans et Ros [1]. En s'y reportant, on sera désagréablement surpris de voir qu'à cette époque 6 échantillons seulement sur 100 étaient irréprochables, 94 étaient anormaux (10 à 11 sont passablas, 17 à 18 sont mauvais, 66 à 67 "présentent des anomalies telles que l'on n'a plus en réalité affaire à du lait, mais plutôt à du pseudo-lait "). Il est bon d'ajouter que certains Iaitiers vendaient $0 \mathrm{fr}$. 40 le litre (et non $0 \mathrm{fr}, 30$ ) du lait qu'ils garantissaient exempts d'eau de mouillage. Or si, d'une façon générale, ce lait était bien supárieur à l'autre, certains de ces échantillons cependant étaient tout à fait défectueux, quelques-uns même inférieurs au lait à $0 \mathrm{fr}$. 30 , alors qu'ils provenaient pourtant de la même étable !

Devant cet état de choses, le Comité de l'Hérault de l'Alliance d'Hygiène sociale créa aussitôt (1907), une "Commission du lait de la Ligue contre la mortalité infantile \%. Cette Commission fut composée de MM. Bernis, inspecteur des Enfants assistés, H. BertinSans, H. Imbert et M. Moye, professeurs à l'Université, Belugou, BLaufus et GaUjoux, docteurs en médecine. Elle travailla activement jusqu'en 1914 et, chaque année, on constata une amélioration nouvelle. On obtint, en outre, la création d'un syndicat des laitiers qui acceptaient la surveillance de leurs produits et se soumettaient aux jugements de la Commission. Malheureusement, tous les laitiers de la ville ou des environs ne firent pas partie de ce syndicat et il est juste de noter que e'était parmi ces dissidents que les condamnations judiciaires furent, et de beaucoup, les plus nombreuses et les plus importantes. 
Depuis 1914, aucune surveillance ne s'est exercée de ce côté. Il paraissait intéressant d'étudier de près la qualité du lait de vache que nous sommes appelés à consommer actuellement. Déjà en 1924, nous avons publié quelques recherches [2] sur la propreté du lait de Montpellier, où nous avons constaté la présence, trop fréquente, de contaminations. En est-il de même au point de vue de la valeur nutritive de ce produit? C'est ce que nous allons essayer de mettre en évidence en comparant nos résultats avec ceux qui ont été publiés antérieurement (avant et pendant la surveillance).

Il existe dans notre ville ou dans ses environs immédiats, 55 laitiers qui approvisionnent nos concitoyens. Mais à ce chiffre, il nous faut ajouter 56 dépôts vendant ce même lait ou celui qui provient de régions assez éloignées (Mazamet par exemple) où la culture permet l'élevage en grand du bétail bovin, soit un total de 11 vendeurs. Sur ce nombre, il nous a été possible d'examiner 100 échantillons provenant de 54 laitiers ou dépositaires choisis absolument au hasard. Dans ces conditions on nous permettra, pensons-nous, d'étendre notre statistique à tout le lait vendu à Montpellier.

Nous n'avons pas voulu étudier ce lait d'une façon absolument complète : nous avons préféré, dans le même temps, examiner un nombre plus considérable d'échantillons. Aussi nous sommes-nous contenté de déterminer seulement:- la densité, la quantité de matière grasse et le point cryoscopique de chacun, estimant que ce sont là des données suffisamment précises et permettant d'apprécier très convenablement les principales fraudes dont ce lait aurait pu être l'objet. Plusieurs fois cependant nous avons cru devoir ajouter une autre notion, l'état d'altération du produit, apprécié par la méthode de la réductasimétrie. Voyons rapidement les méthodes employées et les résultats obtenus.

10 La densité. - Nous l'avons évaluée à l'aide du lacto-densimètre de Quévenne, en rapportant sa valeur à $15^{\circ}$ grâce à des tables spéciales [3]. Elle varie, pour le lait de vache mélangé, de 1,029 à 1,032. Elle s'élève lorsqu'il y a écrémage et s'abaisse si on ajoute de l'eau (mouillage) : on comprend que les deux opérations pratiquées ensemble dans une certaine mesure peuvent laisser une densité normale, ce qui prouve qu'à lui seul, e'est un renseignement notoirement insuffisant. L'étude systématique de la densité de nos échantillons ne nous paraît pas devoir attirer plus longuement l'attention. Les chiffres trouvés pour les laits normaux et rapportés à $15^{\circ}$ oscillent dans les moyennes que nous venons d'indiquer; dans de rares cas, nous avons noté des résultats plus faibles $\left(1,0225\right.$ par exemple $\left.=n^{0} 9\right)$ ou plus forts $\left(1,035=n^{\circ} 3\right)$, mais alors les autres données prouvaient surabondamment qu'il y avait fraude.

$2^{\circ}$ Le dosage du beurre. - Il a été effectué par le procédé de 
Gerber. La quantité de matière grasse contenue dans le lait de vache varie dans de grandes proportions suivant la race de l'animal, sa nourriture, l'âge de son lait, la partie de la traite examinée, etc. Dans ces limites cependant, on peut, pour les laits de mélange, établir la classification suivante, adoptée en 1897 par la Commission municipale d'Etudes de l'alimentation par le lait à Paris :

$1^{\circ}$ Lait riche contenant $50 \mathrm{gr}$. et plus de beurre par litre;

$2^{\circ}$ Lait très bon contenant 45 à $50 \mathrm{gr}$. de beurre par litre;

$3^{\circ}$ Lait bon contenant 40 à 45 gr. de beurre par litre;

$4^{\circ}$ Lait passable contenant 35 à $40 \mathrm{gr}$. de beurre par litre;

$5^{\circ}$ Lait médiocre contenant 30 à 35 gr. de beurre par litre;

$6^{\circ}$ Lais mauvais contenant moins de $30 \mathrm{gr}$. de beurre par litre.

Cependant, il ne faudrait pas croire mouillé ou écrémé tout échantillon de lait rentrant dans les deux dernières catégories (médiocre ou mauvais). En effet, des recherches récentes et inédites entreprises par notre maître, M. le professeur H. IMBERT - que nous remercions tout particulièrement - ont mis en évidence des quantités de matières grasses variant par litre de 17 gr. 74 à 19 gr. 81 chez des vaches bordelaises de dix à douze ans donnant respectivement 10 et 12 litres par jour ; une suisse-hollandaise de sept ans atteignait par contre 50 gr. 44 de beurre et 18 litres de lait quotidiennement! Cela dans une même étable, admirablement tenue du reste, de la région de Mazamet. Les échantillons étaient, bien entendu, pris au pis de la vache et les flacons ¡mmédiatement scellés. Dans le cours de nos analyses, nous avons vu le beurre passer de 20 à 70 gr. par litre. Ce dernier chiffre, qui a été trouvé pour un échantillon dont le $\Delta$ était de $0,50\left(n^{\circ} \quad 9\right)$ e'est-à-dire présentant un mouillage à peu près certain de $10 \%$ environ, ne peut s'expliquer que de la façon suivante: il s'agissait d'un lait prélevé dans une cruche aux trois quarts pleine qui, très probablement, était restée longtemps sans être remuée (peut-être depuis la veille au soir), et dont la crème, en grande partie collectée à la surface, avait été versée en masse dans notre récipient, amenant ainsi pour les elients suivants un véritable écrémage involontaire. Ce qui paraît nous confirmer dans cette idée, e'est le petit nombre de clients servis par cette laiterie. D'ailleurs, sa malpropreté était vraiment excessive et le manque de probité des propriétaires (vérifié par nous-même trois fois sur quatre), les amena maintes fois devant les tribunaux. Nous pourrions faire des observations analogues pour l'échantillon $\mathrm{n}^{\circ} 13$.

$3^{\circ}$ Le point de congélation ou point cryoscopique. - Il a été recherché à l'aide du lacto-cryoscope de Winter, en vérifiant le zéro du thermomètre chaque fois qu'il y avait une ou plusieurs manipulations à pratiquer. Ce point, encore appelé $\Delta$, est beaucoup plus fixe [4] pour le lait de vache que la quantité de matière grasse qui peut y être nor- 
malement contenue. On admet qu'il oseille autour de 0,55 , pour aller, dans les laits de mélange, de 0,532 à 0,586 . Cette recherche est, par elle seule, incapable de donner une notion quelconque sur la quantité de beurre, car l'écrémage ne modifie pas d'une façon appréciable ses résultats. Lorsque le $\Delta$ est normal, il indique simplement que les éléments solubles renfermés dans le lait atteignent leur taux habituel (concentration moléculaire normale). Mais ces sels ne peuvent-ils pas avoir été ajoutés par le fraudeur qui, au lieu de mouiller 1s lait avec de l'eau du robinet, y mettra une solution isotonique? On nous fera peutêtre observer qu'une telle manipulation demande des connaissances particulières, du temps perdu par les tâtonnements et l'emploi d'un cryoscope, appareil d'un prix élevé et d'un usage encore assez délicat. Il n'en est rien. Nous avons pu nous-même, plusieurs fois, en mouillant un lait à $50 \%$ environ avec une solution de sel de cuisine à raison d'une cuillerée à eafé par litre (ce qui correspond aux chiffres de 8 à 9 gr.), ne pas módifier sensiblement son $\Delta$. D'ailleurs, si les laitiers pratiquaient une telle fraude, les laboratoires auraient à leur disposition deux méthodes beaucoup plus précises quoique un peu plus compliquées : la recherche de la constante moléculaire simplifiée ou étude des rapports entre la quantité du lactose et celle du chlorure de sodium contenues dans 1 litre de lait et l'étude de l'indice de réfraction. Ainsi done un $\Delta$ normal ne peut pas, à lui seul, entraîner une' conviction absolue; mais les résultats concordants des trois éléments recherchés présente à nos yeux une réelle valeur. De plus, si le $\Delta$ se rapproçhe par trop de $0^{\circ}$, on peut affirmer que le lait a été mouillé, et l'on peut même, grâce à la formule de Winter, calculer ce mouillage. Si E représente le volume d'eau de mouillage contenue dans le volume $\mathrm{V}$ du lait examiné et $\Delta$ le chiffre trouvé, on peut, d'après cet auteur, écrire :

$$
\frac{\mathrm{E}}{\mathrm{V}}=\frac{0,55-\Delta}{0,55}
$$

C'est de cette formule que nous nous sommes servi pour calculer le mouillage de nos divers échantillons.

Enfin, signalons encore que ce même $\Delta$ peut être très abaissé : c'est ce que nous avons observé deux fois ( $n^{\circ} 99$ et $\left.n^{\circ} 100\right)$. Dans ces cas il ne pouvait s'agir de mouillage. On aurait pu songer à une addition trop forte de $\mathrm{NaCl}$ en vue de masquer la fraude. Il n'en était rien : la recherehe de la constante moléculaire simplifiée nous l'a démontré. Il était encore possible de croire que l'ébullition prolongée avait, par l'évaporation d'une certaine partie de l'eau, accru d'autant la proportion des sels : Nenki et PoDezaski [5] entre autres soutiennent cette opinion. Nous avons done soumis plusieurs échantillons à des temps d'ébullition variables mais couramment employés en cuisine, et jamais nous n'avons noté la moindre modification du $\Delta$ sur ce qu'il 
présentait antérieurement. Nous avons pensé alors que l'altération d'un lait modifiait peut-être son point cryoscopique par suite de la fermentation lactique qui en est la conséquence et nous avons entrepris à ce sujet quelques expériences, confirmées du reste par d'autres auteurs [6], eomme nous avons pu nous en rendre compte ultérieurement.

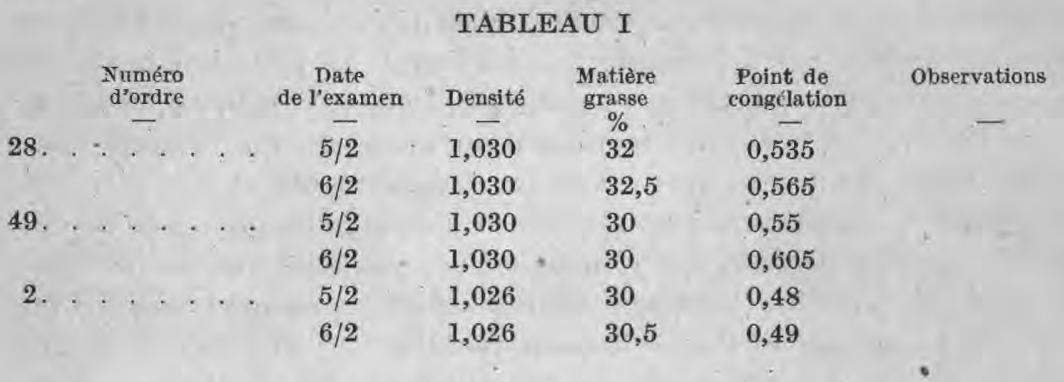

A cause du résultat très positif de ces expériences, nous avons, à partir du mois de mars, cherché à savoir si nos échantillons de lait étaient frais, en complétant tous nos examens par la recherche des réductases.

$4^{\circ}$ La réductasimétrie. - Ce procédé nous a servi, avons-nous dit, à apprécier l'état d'altération de certains faits. On sait qu'il est basé sur la décoloration plus ou moins rapide du bleu de méthyléne par les réductases, probablement microbiennes, contenues dans le lait, qui vieillit. A ce colorant il est bon d'ajouter [7] de la fuchsine qui permet - le mélange donnant au lait une teinte grise - d'apprécier beaucoup plus aisément un virage au rose, plus visible qu'une décoloration du bleu. Et lorsque nous avons été en présence, après nos expériences, d'un deuxième échantillon dont le $\Delta$ était de 0,60 ( $\mathrm{n}^{\circ} 100$ ), nous avons pu très facilement prouver son altération en provoquant son virage rapide au rose, à la température de $40^{\circ}$. A noter également que les échantillons $46,74,77$ et 94 dont le $\Delta$ est normal ou même élevé, sont pauvres en matière grasse. Dans notre statistique, nous les considérerons comme falsifiés (mouillés ou écrémés) et nous admettrons que leur point cryoscopique est dû à leur altération ; nous l'admettrons, quoique n'ayant pu le vérifier, puisque, à cette date, nous ne recherchions pas systématiquement la présence des réductases dans nos laits. Mais si l'altération d'un lait est capable d'abaisser son point cryoscopique, on pourra nous objecter que tous nos résultats sont entachés d'erreur. Il n'en est rien. D'abord il arrive assez souvent qu'un lait conservé vingt-quatre heures au laboratoire ne voit son $\Delta$ que très faiblement abaissé $(0,54$ à 0,55 par exemple pour le numéro 2$)$; un autre demeurera ce qu'il était la veille. Et puis nous avons surtout 
opéré en saison froide, et, plus tard, nous avons toujours pris nos précautions pour que le lait soit étudié immédiatement après son achat, et nous avons recherché systématiquement, comme e'est indiqué plus haut, son degré d'altération. Dans ces conditions, nos laits (sauf les numéros que nous venons de signaler) étaient frais et nos conclusions doivent être considérées comme conformes à la réalité. Du reste, pour les deux laits à $\Delta$ trop élevés, la densité et le dosage de la matière grasse ont montré des chiffres normaux et nous avons vu que ces deux éléments ne sont guère modifiables, eux, par la fermentation lactique.

$5^{\circ}$ Enfin, nous avons appliqué plusieurs fois une méthode que nous avons récemment proposée avec M. CASTAGNÉ [8] afin d'apprécier facilement si un lait, auparavant reconnu non mouillé, a été ou non écrémé. Il s'agit simplement, sur une grosse goutte de l'échantillon de lait à examiner, de faire tomber une petite goutte de sérum additionné d'encre de Chine dans la proportion de 1 pour 15 de sérum. $\mathrm{Si}$ l'on n'a pas de sérum frais, on peut employer du sérum thérapeutique, même ancien. Lorsque le lait est très écrémé, il se forme, en trente à quarante-cinq secondes, des traînées noires partant du centre et représentant assez bien une fleur de romposée radiée. Lorsque le lait n'a pas subi d'écrémage - si on ne l'a privé que d'une faible partie de ses matières grasses - l'expérience donne l'image d'un œil de verre. Disons tout de suite que, vus de cet angle, nos échantillons nous ont paru en général n'avoir pas été écrémés. C'ést, du reste, ce que prouvent les examens pratiqués par le Laboratoire de la Répression des fraudes qui, en 1925, sur 473 prélèvements effectués n'a trouvé qu'un seul écrémage à $35 \%$ environ, et, dans les six premiers mois de 1926, n'a infligé qu'une seule amende pour une fraude analogue sur 278 échantillonsexamin

Telles sont les méthodes dont nous nous sommes servi. Avant de donner les résultats de nos déterminations, et pour bien faire, saisir la différence de la fraude dans les trois périodes dont nous avons parlé plus haut, nous nous permettrons de donner deux tableaux, relevés dans les travaux de MM. Bertin-Sans et de son élève Ros [1].

\section{TABLEAU II}

Avant tout contrôle (1906), (lait à 0 fr. 30 le litre).

Classement d'après la formule de Winter des divers laits examinés.

1 ou $1,75 \%$ a un $\Delta$ de 0,28 eorrespondant à un mouillage de $49 \%$.

5 ou $8,75 \%$ ont un $\Delta$ de $0,37-0,38$ correspondant à un mouillage de $32-30 \%$.

4 ou $7 \%$ ont un $\Delta$ de $0,39-0,41$ correspondant à un mouillage de $29-25 \%$.

8 ou $14 \%$ ont un $\Delta$ de $0,42-0,44$ correspondant à un mouillage de $24-20 \%$.

15 ou $26,25 \%$ ont un $\Delta$ de $0,445-0,465$ correspondant à un mouillage de $19-15 \%$.

17 ou $29,75 \%$ ont un $\Delta$ de $0,47-0,495$ correspondant à un mouillage de $14-10 \%$.

4 ou $7 \%$ ont un $\Delta$ de $0,50-0.52$ correspondant à un mouillage de $9-5 \%$.

3 ou $5,25 \%$ ont un $\Delta$ de $0,54-0,55$ correspondant à un mouillage de $0 \%$. 


\section{TABLEAU III}

Pendant le contrôle (1908).

(Ici deux séries de recherches portant sur les laits surveillés et sur les laits non surveillés, c'est-à-dire provenant de dépots de revente ou livrés par les laitiers non syndiqués.)

Degré de mouillage. - Proportion pour 100.

Echantillons non mouillés . . . . . . . . 37

\begin{tabular}{|c|c|c|}
\hline 1906 & & 1908 \\
\hline $\begin{array}{l}\text { Lait à } 0,40 \text { le litre } \\
\text { avant la } \\
\text { surveillance }\end{array}$ & $\begin{array}{l}\text { Lait } \\
\text { sur- } \\
\text { veillé }\end{array}$ & $\begin{array}{l}\text { Lait de dépôt } \\
\text { non } \\
\text { surveillé }\end{array}$ \\
\hline 37 & 81,5 & 40 \\
\hline 7,4 & 9,5 & 16 \\
\hline 14,8 & 5 & 32 \\
\hline 22,2 & 0 & 12 \\
\hline 18,5 & 0,3 & 0 \\
\hline
\end{tabular}

Voyons maintenant le résultat de nos expériences:

TABLEAU IV

Résultats de nos recherches (1926)

\begin{tabular}{|c|c|c|c|c|c|}
\hline $\begin{array}{l}\text { Numéro } \\
\text { d'ordre }\end{array}$ & $\begin{array}{l}\text { Date de } \\
\text { l'examen }\end{array}$ & Densité & $\begin{array}{l}\text { M. G. } \\
\text { (gr.p.litre) }\end{array}$ & $\begin{array}{l}\text { Point de } \\
\text { congélation }\end{array}$ & $\begin{array}{l}\text { Observations } \\
\text { (Laits de même provenance) }\end{array}$ \\
\hline 1. . & $1 / 6$ & 1,0275 & 30 & 0,47 & V. $n^{\circ s} 28$ et 78 . \\
\hline 2. & $5 / 2$ & 1,026 & 30 & 0,48 & V. $n^{\text {os }} 9,15$ et 46. \\
\hline 3. & $28 / 1$ & 1,035 & 20 & 0,49 & \\
\hline 4. & $5 / 3$ & 1,0285 & 28 & 0,49 & \\
\hline 5. & $1 / 6$ & 1,0306 & 33 & 0,49 & V. $\mathrm{n}^{\circ \mathrm{s}} 10,11,26$ et 55 . \\
\hline 6. & $12 / 3$ & 1,029 & 28 & 0,50 & \\
\hline 7. . & $22 / 3$ & 1,030 & 29 & 0,50 & V. $\mathrm{n}^{\circ \mathrm{s}} 14$ et 61 \\
\hline 8. & $22 / 3$ & 1,030 & 31 & 0,50 & V. $n^{0 s} 16,23$ et 41 . \\
\hline 9. & $24 / 2$ & 1,0225 & 70 & 0,50 & V. $n^{08} 2,15$ et 46 \\
\hline 10. & $19 / 3$ & 1,029 & 31 & 0,51 & V. $n^{0 s} 5,11,26$ et 55 \\
\hline 11. & $8 / 3$ & 1,030 & 31 & 0,51 & V. $\mathrm{n}^{\mathrm{os}} 5,10,26$ et 55 \\
\hline 12. & $18 / 3$ & 1,029 & 35 & 0,51 & V. $\mathrm{n}^{\text {os }} 40,84$ et 87 , \\
\hline 13. & $9 / 2$ & 1,025 & 52 & 0,515 & \\
\hline 14. . & $31 / 5$ & 1,023 & 28 & 0,52 & V. $\mathrm{n}^{\text {os }} 7$ et 61 . \\
\hline 15. & $26 / 3$ & 1,029 & 32 & 0,52 & V. $\mathrm{n}^{\text {os }} 2,9$ et 46 . \\
\hline 16. & $1 / 6$ & 1,0285 & 32 & 0,52 & V. $n^{0 s} 8,23$ et 41 . \\
\hline 17. & $25 / 1$ & 1,030 & 32,5 & 0,52 & V. $\mathrm{n}^{\circ \mathrm{s}} 35,37$ et 82 \\
\hline 18. & $23 / 2$ & 1,031 & 39 & 0,52 & V. nos 53 et 67 . \\
\hline 19. & $5 / 2$ & 1,031 & 34 & 0,525 & \\
\hline 20. & $30 / 1$ & 1,031 & 38 & 0,525 & \\
\hline 21. & $20 / 2$ & 1,030 & 30 & 0,53 & \\
\hline 22. & $27 / 1$ & 1,0295 & 30 & 0,53 & V. $\mathrm{n}^{0} 58$. \\
\hline 23. & $13 / 4$ & 1,030 & 30,5 & 0,53 & V. nos 8,16 et 41 . \\
\hline 24. & $30 / 1$ & 1,0305 & 31 & 0,53 & V. $n^{0} 88$. \\
\hline
\end{tabular}




\begin{tabular}{|c|c|c|c|c|c|c|c|}
\hline $\begin{array}{l}\text { Num } \\
\text { d'or }\end{array}$ & & & $\begin{array}{l}\text { Date de } \\
\text { l'examen }\end{array}$ & Densité & $\begin{array}{l}\text { M. G. } \\
\text { (gr.p.litre) }\end{array}$ & $\begin{array}{l}\text { Point de } \\
\text { congélation }\end{array}$ & $\begin{array}{l}\text { Observations } \\
\text { (Laits de même provenance) }\end{array}$ \\
\hline 25. . & - & . & $22 / 3$ & 1,030 & 31,5 & 0,53 & V. nos 29 et 32 . \\
\hline 26. . & . & , & $1 / 3$ & 1,028 & 34 & 0,53 & V. $\mathrm{n}^{\circ \mathrm{s}} 5,10,11$ et 55 \\
\hline 27. & . & . & $12 / 3$ & 1,0295 & 38 & 0.53 & V. no 98 \\
\hline 28. . & . & . & $5 / 2$ & 1,030 & 32 & 0,535 & V. $n^{\text {os }} 1$ et 78. \\
\hline 29. . & . & . & $25 / 1$ & 1,028 & 35 & 0,535 & V. nos 25 et 32 . \\
\hline 30. . & . & . & $12 / 2$ & 1,029 & 32 & 0,54 & V. nos $^{\circ} 31,59$ et 75 . \\
\hline 31 . . & . & . & $6 / 3$ & 1,030 & 32 & 0,54 & V. nos $^{\circ 0,} 59$ et 75 . \\
\hline 32. . & . & . & $1 / 3$ & 1,029 & 32 & 0,54 & V. nos 25 et 29 . \\
\hline 33. . & . & . & $8 / 3$ & 1,029 & 34 & 0,54 & \\
\hline 34. . & . & . & $29 / 5$ & 1,030 & 34 & $0, \check{5} 4$ & V. nos 56,68 et 97 . \\
\hline 35. & . & ; & $3 / 2$ & 1,030 & 34,50 &, 54 & V. $n^{\circ \mathrm{s}} 17,37$ et 82 \\
\hline 36. . & . & - & . $28 / 5$ & 1,030 & 35 & 0,54 & V. no 72 , \\
\hline 37. . & . & . & $26 / 5$ & 1,031 & 35 & 0,54 & V. $n^{\circ s} 17,35$ et 82 . \\
\hline 38. : & . & . & $8 / 3$ & 1,030 & 36 & 0,54 & \\
\hline 39. . & . & . & $6 / 2$ & 1,032 & 41 & 0,54 & \\
\hline 40 . . & . & . & $5 / 3$ & 1,031 & 42 & 0,54 & V. nos 12,84 et 87 . \\
\hline 41. . & . & . & $28 / 1$ & 1,030 & 59 & 0,54 & V. $\mathrm{n}^{\text {os }} 8,16$ et 23 \\
\hline 42. & . & . & $28 / 1$ & 1,0315 & 31 & 0,545 & V. $n^{\circ} 48$. \\
\hline 43. . & . & . & $23 / 2$ & 1,029 & 32 & 0,545 & \\
\hline 44. . & . & . & $2 / 3$ & 1,030 & 37 & 0,545 & \\
\hline 45. . & . & . & $27 / 1$ & 1,0315 & 38 & 0,545 & 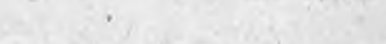 \\
\hline 46. . & . & . & $9 / 2$ & 1,0315 & 27 & 0,55 & V. nos 2,9 et 15 \\
\hline 47. . & . & . & $10 / 3$ & 1,033 & 28 & 0,55 & V. $\mathrm{n}^{\circ \mathrm{s}} 51$ et 60 \\
\hline 48. . & . & . & $28 / 1$ & 1,0315 & 30 & 0,55 & V. no 42 . \\
\hline 49. & . & . & $5 / 2$ & 1,030 & 30 & 0,55 & V. no 99 . \\
\hline 50. : & . & . & $23 / 3$ & 1,0295 & 30,5 & 0,55 & V. no 76 \\
\hline 51. : & . & , & $25 / 3$ & 1,029 & 31 & 0,55 & V. nos 47 et 60 . \\
\hline 52. . & . & . & $29 / 1$ & 1,029 & 31 & 0,55 & \\
\hline 53. & . & . & $26 / 1$ & 1,0295 & 31 & 0,55 & V. nos 18 et 67 \\
\hline 54. & . & . & $27 / 2$ & 1,030 & 31,5 & 0,55 & V. $\mathrm{n}^{\text {os }} 73,79,80,86$ et 8 \\
\hline 55. & , & . & $24 / 3$ & 1,030 & 32 & 0,55 & V. $\mathrm{n}^{\text {os }} 5,10,11$ et 26 \\
\hline 56. & $\because$ & . & $23 / 4$ & 1,032 & 32 & 0,55 & V. nos 34,68 et 97. \\
\hline 57. . & . & . & $20 / 2$ & 1,030 & 33 & 0,55 & $x_{1}=y^{\prime}$ \\
\hline 58. . & . & . & $24 / 2$ & 1,030 & 33 & 0,55 & V. $n^{0} 22$ \\
\hline 59. . & . & . & $31 / 5$ & 1,028 & 34 & 0,55 & V. $\mathrm{n}^{\mathrm{os}} 30,31$ et 75 . \\
\hline 60. . & . & . & $15 / 4$ & 1,029 & 34 & 0,55 & V. nos 47 et 51 . \\
\hline 61. . & . & . & . $24 / 2$ & 1,029 & 34 & 0,55 & V. nos 7 et 14 \\
\hline 62. & . & . & $27 / 1$ & 1,0325 & 34 & 0,55 & \\
\hline 63. . & . & . & $25 / 1$ & 1,030 & 35 & 0,55 & \\
\hline 64. . & . & . & $26 / 1$ & 1,029 & 35 & 0,55 & \\
\hline 65. . & . & . & $5 / 3$ & 1,0305 & 36 & 0,55 & \\
\hline 66. & . & . & $12 / 2$ & 1,0305 & 36 & 0,55 & V. no 83 . \\
\hline 67. . & . & . & $15 / 3$ & 1,031 & 36 & 0,55 & V. nos 18 et 53 \\
\hline 68. . & . & . & $15 / 3$ & 1,029 & 37 & 0,55 & V. $\mathrm{n}^{\text {os }} 34,56$ et 97 . \\
\hline 69. . & . & . & $12 / 3$ & 1,030 & 37 & 0,55 & \\
\hline 70. & . & . & $2 / 3$ & 1,030 & 37 & 0,55 & \\
\hline
\end{tabular}




\begin{tabular}{|c|c|c|c|c|c|c|c|}
\hline $\begin{array}{l}\text { Num } \\
\text { d'or }\end{array}$ & & & $\begin{array}{l}\text { Date de } \\
\text { l'examen }\end{array}$ & De:usité & $\begin{array}{l}\text { M. G. } \\
\text { (gr. p. litre) }\end{array}$ & $\begin{array}{l}\text { Point de } \\
\text { congélation }\end{array}$ & $\begin{array}{c}\text { Observations } \\
\text { (Laitis de même provenance) }\end{array}$ \\
\hline 71. . & 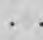 & & $29 / 5$ & 1,030 & 37 & 0,55 & \\
\hline 72. . & . . & - & $2 / 3$ & 1,031 & 39 & 0,55 & V. $\mathrm{n}^{\circ} 36$. \\
\hline 73. . & . . & . & $28 / 1$ & 1,0305 & 44 & 0,55 & V. $\mathrm{n}^{0 \mathrm{~s}} 54,79,80,86 \mathrm{e}$ \\
\hline 74. . & . &  & $20 / 2$ & 1,030 & 28 & 0,555 & \\
\hline 75. . & . . & . & $17 / 3$ & 1,030 & 30 & 0,555 & V. nos 30,31 et 59 . \\
\hline 76. . & . . & - & $27 / 2$ & 1,0315 & 43,5 & 0,555 & V. $\mathrm{n}^{\circ} 50$ \\
\hline 77. . & . . & . & $20 / 2$ & 1,030 & 28 & 0,56 & \\
\hline 78. . & . & . & $20 / 4$ & 1,030 & 30 & 0,56 & V. $n^{0 s} 1$ et 28. \\
\hline 79. . & . & - & $16 / 4$ & 1,030 & 30 & 0,56 & $V \cdot n^{0 s} 54,73,80,86$ et \\
\hline 80. . & . & . & $27 / 5$ & 1,029 & 32 & 0,56 & V. $n^{\text {os }} 54,73,79,86$ et \\
\hline $81 .$. & . & . & $26 / 5$ & 1,031 & 33 & 0,56 & \\
\hline 82. . & . & . & $22 / 4$ & 1,030 & 34 & 0,56 & V. $\mathrm{n}^{0 \mathrm{~s}} 17,35$ et 37 . \\
\hline 83. . & . & . & $15 / 3$ & 1,031 & 34 & 0,56 & V. $n^{\circ} 66$ \\
\hline 84. . & . & . & $31 / 5$ & 1,028 & 34 & 0,56 & V. nos 12,40 et 87 . \\
\hline 85. . & . & & $1 / 3$ & 1,032 & 36 & 0,56 & \\
\hline 86. & . & e & $16 / 3$ & 1,030 & 36 & 0,56 & V. $\mathrm{n}^{\mathrm{os}} 54,73,79,80$ \\
\hline 87. . & . & . & $9 / 2$ & 1,031 & 37 & 0,56 & V. nos 12,40 et 84 . \\
\hline 88. . & . & . & $6 / 3$ & 1,0315 & 38 & 0,56 & V. $n^{\circ} 24$ \\
\hline 89. . & . & . & $10 / 3$ & $1 \cdot 030$ & 46 & 0,56 & V. $\mathrm{n}^{\mathrm{os}} 54,73,79,80$ \\
\hline 90. . & . & . & $30 / 1$ & 1,030 & 30 & 0.565 & v. $n^{\circ s} 95$ et 100 . \\
\hline 91. . & . & . & $26 / 4$ & 1,030 & 35 & 0,565 & \\
\hline 92. . & . & . & $29 / 1$ & 1,029 & 47 & 0,565 & \\
\hline 93. . & . & . & $6 / 3$ & 1,031 & 32,5 & 0,57 & \\
\hline 94. . & . & . & $12 / 2$ & 1,034 & 28 & 0,58 & \\
\hline 95. . & - & 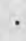 & $28 / 4$ & 1,030 & 30 & 0,58 & V. $n^{\text {os }} 90$ et 100. \\
\hline 96. . & . & & $23 / 2$ & 1,030 & 33 & 0,58 & \\
\hline 97. & . & . & $27 / 2$ & 1,0295 & 34 & 0,58 & V. nos 34,56 et 68 . \\
\hline 98. . & . & & $25 / 1$ & 1,029 & 39 & 0,58 & V. $n^{0} 27$. \\
\hline 99: . & - & & $5 / 3$ & 1,030 & 34 & 0,60 & V. $\mathrm{n}^{\circ} 49$. \\
\hline 00. . & . & & $10 / 3$ & 1,030 & 37 & 0,60 & V. $\mathrm{n}^{\mathrm{os}} 90$ et 95. \\
\hline
\end{tabular}

Si l'on veut résumer cette série de chiffres un peu trop longue et peut-être difficile à lire, nous pourrons, en imitant le tableau no III auquel nous renvoyons encore, dire que nous avons relevé à Montpellier en 1926 :

\section{TABLEAU $V$}

Echantillons non mouillés . . . . . . . . . . $67 \%$

Echantillons mouillés à moins de $5 \%$. . . . . . 13 -

Echantillons mouillés de 5 à $10 \%$........ 15 -

E'chantillons mouillés de 10 à $15 \%$....... 5 -

Echantilons mouillés a plus de $15 \%$....... 0 -

Cette heureuse amélioration d'un ancien état de choses vraiment regrettable, étant donnée la valeur alimentaire du produit, était, nous semble-t-il, à faire connaître. Quant à sa cause nous ne pouvons 
que la mettre sur le compte des travaux importants poursuivis inlassablement de 1907 à 1914 par la "Commission du Lait ». Il est justice de rendre à ses membres ce qui leur est dû. L'activité du service de la répression des fraudes peut aussi prendre sa part de cet heureux résultat.

Conclusions. - Comme on peut s'en rendre compte en examinant les résultats de nos recherches et en les comparant à ceux que d'autres ont apportés antérieurement :

$1^{\circ}$ Le lait consommé actuellement à Montpellier est, en général, d'une bonne qualité ;

$2^{\circ}$ Par contre il a été très mauvais avant 1907 ;

$3^{\circ} \mathrm{Il}$ s'est amélioré progressivement sous l'influence de la "Commission de Surveillance " (1907-1914), dont l'effet semble se poursuivre encore aujourd'hui ;

$4^{0} \mathrm{Si}$ ce même lait est trop souvent sale ou très sale, et très riche en germes, cela semble provenir, non d'une mauvaise volonté, mais, chez nos laitiers, d'un manque d'éducation, d'une ignorance que l'on pourrait aisément corriger par des conseils pratiques, analogues à ceux que nous proposions en 1924, c'est-à-dire :

a) D'abord la propreté des vaches, des étables, des récipients et des trayeurs;

b) Le lait devrait ensuite être filtré aussitôt après sa récolte ;

c) Enfin le lait devrait être conservé à la glacière, ou, du moins, au froid jusqu'au moment de la vente.

\section{BIBLIOGRAPHIE}

[1] Bertin-SAns et Ros, Montpellier Médical, 11 mars et $1^{\mathrm{er}}$ avril 1906.

[2] Carriev, Languedoc Médical, 10 mai 1924.

[3] VIlliens et Colins, Altération et falsification des substances alimentaires, p. 589.

[4] Voir la critique souvent acerbe de cette méthode dans la thèse de VILLEJEAN $\mathrm{n}^{\circ} 25$, Paris, 1905.

[5] Nenki et Podezaski (analysé in Annales de Chimie analytique, p. 275, 1904).

[6] Winter et Parmentier, Revue générale du Lait, Bruxelles, 1904.

[7] Bertin-Sans et Gaujoux, Revue d'Hygiène et de Police sanitaire, p. 866, sept. 1909 et p. 258, mars 1914, qui donne la bibliographie de la question.

[8] Carrieu et Castagnf́, Le Lait, p. 188-192, 1924. 